\title{
An Investigation of Factors Involved in Hall Thruster Wall Erosion Modeling
}

\author{
John T. Yim, Michael Keidar \\ University of Michigan, Ann Arbor, MI 48109, USA
}

\begin{abstract}
A hydrodynamic description of the plasma flow within a SPT-100 type Hall thruster is used to study the erosion of the channel walls over time. The simulation of thousands of hours of erosion is performed on the order of tens of minutes of computation time. The calculated erosion matches satisfactorily against experimental data, especially the erosion at the exit plane. The effects of scattering collisions, ion velocity distributions, sputtering threshold energy, sputter yield curve fits, and potential drop within the thruster on the erosion are examined. While all of the above do affect erosion rates, the semi-empirical sputter yield curve fits, especially at the low ion energies prevalent in Hall thrusters, play the largest role in influencing the simulated erosion of the channel walls.
\end{abstract}

\section{Nomenclature}

$\begin{array}{ll}B & \text { Magnetic field }[\mathrm{T}] \\ E & \text { Electric field }[\mathrm{V} / \mathrm{m}] \\ Q & \text { Heat (power density) }\left[\mathrm{W} / \mathrm{m}^{3}\right] \\ T & \text { Temperature }[\mathrm{K}] \\ V & \text { Velocity }[\mathrm{m} / \mathrm{s}] \\ a & \text { Sound speed }[\mathrm{m} / \mathrm{s}] \\ e & \text { Elementary charge }[\mathrm{C}] \\ j & \text { Current density }\left[\mathrm{A} / \mathrm{m}^{2}\right] \\ k & \text { Boltzmann constant }[\mathrm{J} / \mathrm{K}] \\ m & \text { Particle mass }[\mathrm{kg}] \\ n & \text { Number density }\left[\mathrm{m}^{-3}\right] \\ p & \text { Pressure }\left[\mathrm{N} / \mathrm{m}^{2}\right] \\ t & \text { Time }[\mathrm{s}] \\ \beta & \text { Ionization rate }\left[\mathrm{m}^{3} / \mathrm{s}\right] \\ \mu & \text { Mobility }\left[\mathrm{m}^{2} /(\mathrm{V} \mathrm{s})\right] \\ \nu & \text { Collision frequency }\left[\mathrm{s}^{-1}\right] \\ \rho & \text { Density }\left[\mathrm{kg} / \mathrm{m}^{3}\right] \\ \text { Subscript } \\ a & \text { Neutral atom } \\ e & \text { Electron } \\ i & \text { Ion } \\ r & \text { Radial } \\ z & \text { Axial }\end{array}$

${ }^{*}$ Graduate Student, Department of Aerospace Engineering, AIAA Student Member.

${ }^{\dagger}$ Assistant Research Scientist, Department of Aerospace Engineering, AIAA Senior Member.

${ }^{\ddagger}$ Professor, Department of Aerospace Engineering, AIAA Associate Fellow. 


\section{Introduction}

s lifetime requirements desired for Hall thruster operations increase, there is a greater need to be able Ato predict and analyze the wear of the thruster over time. The main mechanism of Hall thruster failure is the erosion of the acceleration channel walls to the point where the magnetic coils are exposed to the plasma flow. Experimental testing to determine the rate and extent of wall erosion is expensive and time consuming. Thus, capturing the erosion process through computational simulations is a useful means of predicting lifetime for design and analysis purposes alongside experimentally obtained data.

Interest in Hall thruster erosion, including the development of Hall thruster erosion models, has been increasing recently. ${ }^{1-3}$ However, there are still aspects of the erosion process that remain unsatisfactorily modeled. In our previous work, we developed a hydrodynamic-based erosion model for Hall thrusters. ${ }^{4,5}$ However, that model was unable to capture the low erosion rates registered at later portions of the thruster life after around 1000 hours and instead calculated zero erosion. The work presented in this paper presents the same sheath and plasma model, though with a new numerical scheme, with investigations on various aspects of the plasma flow and sputter model that are thought to perhaps contribute to the erosion process.

It is thought that perhaps a secondary mechanism for erosion may dominate at the later times. ${ }^{6}$ Thus, the contribution to erosion from scattering collisions is examined. Also, the sputtering portion of the model is further investigated with the incorporation of ion velocity distributions instead of assuming monoenergetic streams. The extent of the influence of different empirical sputter yield fits on the erosion rates is also observed. Finally, a simple parametric study of the effects of changing the plasma parameters is performed.

The numerical scheme for the hydrodynamic model is different than the one we have used before and is described in the next section. The results of the various test cases are compared to each other and experimental data. Discussion of the results and areas of further work are given later in the paper.

\section{Model}

\section{A. Plasma model}

A hydrodynamic description of the quasi-neutral plasma flow within the thruster is used. The ions are modeled using a finite-volume flux-splitting method on an axisymmetric Cartesian mesh. ${ }^{7}$ The isothermal ion equations can be written in conservative form as

$$
\begin{gathered}
\frac{\partial \rho}{\partial t}+\frac{\partial\left(\rho V_{z}\right)}{\partial z}+\frac{\partial\left(\rho V_{r}\right)}{\partial r}=\beta n_{a} \rho \\
\frac{\partial\left(\rho V_{z}\right)}{\partial t}+\frac{\partial\left(\rho a^{2}+\rho V_{z}^{2}\right)}{\partial z}+\frac{\partial\left(\rho V_{z} V_{r}\right)}{\partial r}=e n E_{z}+\beta n_{a} V_{a} \rho \\
\frac{\partial\left(\rho V_{r}\right)}{\partial t}+\frac{\partial\left(\rho V_{z} V_{r}\right)}{\partial z}+\frac{\partial\left(\rho a^{2}+\rho V_{r}^{2}\right)}{\partial r}=e n E_{r}
\end{gathered}
$$

The source terms that remain on the right hand side of the above equations result from the effects of ionization and the electric field. ${ }^{8}$ The components of the electric field are calculated from the electron momentum equation,

$$
\nabla \cdot\left(\rho \vec{V}_{e} \vec{V}_{e}\right)=-\nabla p_{e}-e n\left(\vec{E}+\vec{V}_{e} \times \vec{B}\right)-\rho_{e} \nu_{e} \vec{V}_{e}
$$

The electrons are assumed to be inertia-less and mainly constrained along the magnetic field lines, which for simplicity vary only in the axial direction. Then, the electron pressure term can be folded into the conservative side of the ion momentum equations, Eqs. (2) and (3), through a new effective sound speed defined by

$$
a^{*}=\sqrt{a^{2}+\frac{k T_{e}}{m_{i}}}
$$

The ion momentum equations then become

$$
\begin{gathered}
\frac{\partial\left(\rho V_{z}\right)}{\partial t}+\frac{\partial\left(\rho\left(V_{z}^{2}+a^{* 2}\right)\right)}{\partial z}+\frac{\partial\left(\rho V_{z} V_{r}\right)}{\partial r}=\frac{j_{e_{z}}}{\mu_{e}}+\beta n_{a} V_{a} \rho \\
\frac{\partial\left(\rho V_{r}\right)}{\partial t}+\frac{\partial\left(\rho V_{z} V_{r}\right)}{\partial z}+\frac{\partial\left(\rho\left(V_{r}^{2}+a^{* 2}\right)\right)}{\partial r}=0 \\
2 \text { of } 9
\end{gathered}
$$


The electron mobility is dependent on the effective electron collision frequency which is a sum of the collision frequencies of electrons with the ions, neutral atoms, and the walls. It also includes anomalous Bohm diffusion in the form of

$$
\nu_{B}=\alpha_{B} \omega_{e}
$$

where $\alpha_{B}$ is the coefficient for the Bohm diffusion, here set to $1 / 35$, and $\omega_{e}$ is the electron gyrofrequency.

The system of equations is closed with the electron temperature calculated from an electron energy equation,

$$
\frac{3}{2} \frac{\partial\left(j_{e} T_{e}\right)}{\partial z}=Q_{\text {joule }}-Q_{\text {ion }}-Q_{\text {wall }}-Q_{\text {el }}
$$

that balances Joule heating with losses due to ionization, wall collisions, and elastic relaxation. More details of these equations are described elsewhere. ${ }^{5}$

A Roe solver is used to calculate the fluxes across each of the cell interfaces, which in turn are used to update the cell values at each time step. ${ }^{9}$ The process is iterated until the simulation converges to a representative state. Since the erosion process occurs on a much longer time scale than the plasma oscillations that are present within the thruster, an averaged representative state can be used for the erosion calculations.

As the walls erode away, a cut cell method is employed to better model the changing geometry. ${ }^{10,11}$ This provides the benefit of using the same Cartesian mesh at each time step of the simulation instead of having to generate a new mesh every time as the wall boundaries change. The cells that the walls pass through are 'cut' accordingly and are given proper boundary conditions to account for the local wall angle. Cell sections or corners that are cut too small are merged with bordering cells so the time step does not become unnecessarily small in fulfilling the CFL condition. Figure 1 displays examples of cut and merged cells. The actual boundaries of the simulation are set at the sheath edge instead of the actual wall boundary since several of the assumptions in the governing equations break down within the sheath. The properties of the sheath, which also affect the erosion, are described in earlier work. ${ }^{8}$

The model is run for a SPT-100 type thruster for which experimental erosion data exists. Table 1 lists the overall geometry and parameters used for the simulations. A prescribed axial profile of the radial magnetic field is used in addition to the other inputs. The computational mesh used for the results presented here is 96 cells by 122 cells. 4000 hours of thruster erosion time are simulated with 10 hour time steps between each update of the wall profiles. The wall clock time required to run this model on a desktop computer is on the order of tens of minutes.

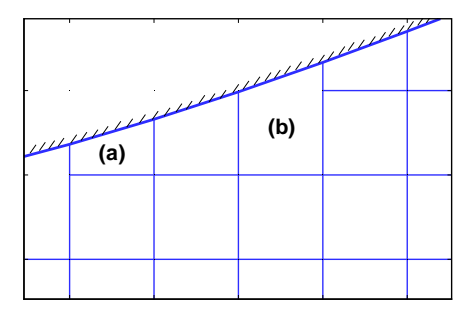

Figure 1. Examples of (a) cut and (b) merged cells.

\begin{aligned} & \hline \hline Parameter Value \\ & \hline outer diameter: $0.1000 \mathrm{~m} \\ &$ inner diameter: $0.0686 \mathrm{~m} \\ &$ channel length: $0.024 \mathrm{~m} \\ &$ mass flow rate: $4.9 \times 10^{-6} \mathrm{~kg} / \mathrm{s} \\ &$ discharge voltage: $300 \mathrm{~V} \\ &$ discharge current: $4.5 \mathrm{~A} \\ &$\hline \hline\end{aligned}

Table 1. Input parameters used for an SPT-100 thruster.

\section{B. Erosion model}

An empirical model is used to obtain the sputter yields from the ion current to the walls. Experiments have been conducted where xenon ion beams have been directed onto boron nitride samples. ${ }^{12,13}$ Dependency of the sputter yield on both angle and energy of incidence have been reported and are shown in Figs. 2 and 3. Garnier et al. do not give specific values for error bars, but report the values are accurate within 20\%, which is what is shown in the figures. The energy dependent fit to the data from Britton et al. is applied to the data points at 300 and $600 \mathrm{~V}$. For the angular dependence, the data from Garnier are normalized to the values obtained with the ion beam at normal incidence, while the data from Britton are normalized at the values taken at 15 degrees from the wall normal. Even taking all of this into account, it can be seen 
that there is a non-trivial amount of uncertainty in the sputter yield data. The sputter yields measured by Britton are higher overall compared to Garnier but the angular dependence is less pronounced.

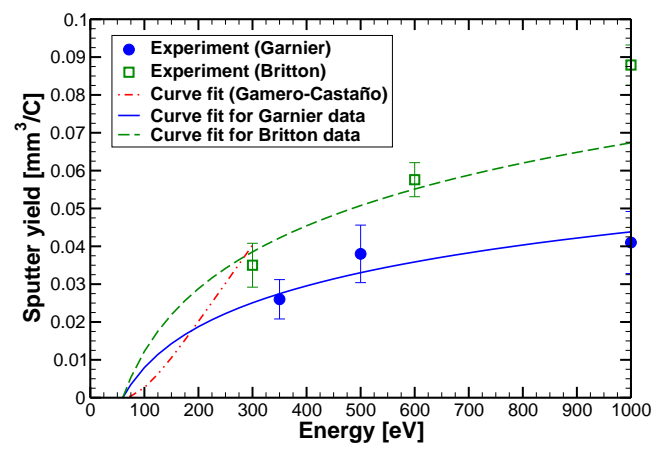

Figure 2. The sputter yield dependence on ion energy.

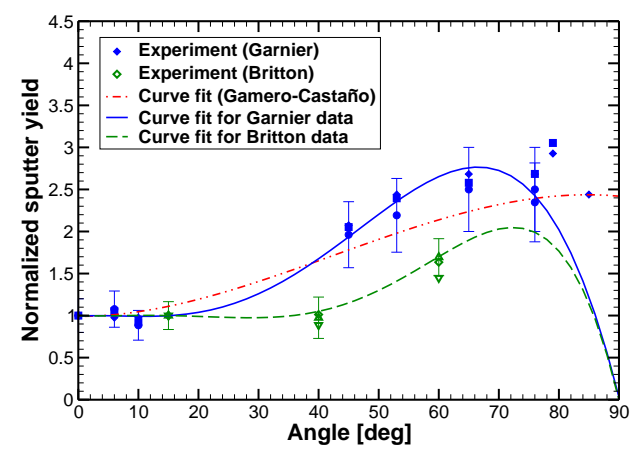

Figure 3. The normalized sputter yield dependence on incident angle.

At low energies, below $300 \mathrm{eV}$, no experimental sputter yield data exists. This is especially unfortunate as most ions in the SPT-100 will have energies in this range since the total discharge voltage of the thruster is $300 \mathrm{~V}$. The threshold energy required for sputtering is also unknown. Thus, for the present modeling efforts, a threshold energy is assumed and the data is extrapolated from measured values. Three curve fits are presented in Figs. 2 and 3. Two are logarithmic fits applied to the Garnier and Britton data with a $60 \mathrm{eV}$ threshold value applied. The third, provided by Gamero-Castaño et al. ${ }^{3}$ for the $\mathrm{BNSiO}_{2}$ sputter data also given by Garnier, is shown in comparison as well. They use a modified form of Yamamura and Tawara's ${ }^{14}$ empirical formula for sputtering of monatomic solids. Each of the three fits are used to observe the magnitude of the effect of the sputter model on the overall erosion of the thruster walls.

In previous work, for the calculation of the erosion rate, monoenergetic ion streams were used at each location along the walls. In actuality, however, it can be expected that there is a distribution of incident angles and energies. A Maxwellian distribution based on the ion temperature is used for the axial and radial components of the ion velocities. This creates a distribution of incident angles and impact energies that are taken into account in the sputter yield calculations. A Maxwellian distribution for the ions is not unreasonable as the neutral atoms from which they are created are likely to be close to a Maxwellian distribution. Due to the low collision rate between the ions and electrons, the velocity distributions as well as the ion temperature are not expected to change significantly within the channel.

Another contributor to erosion is from ions scattered from the main flow to the walls by elastic collisions with the neutral atoms. ${ }^{15}$ The ions that are deflected to the walls may not have impacted the walls otherwise, and thus this adds to the overall erosion. The collision rate is calculated from a cross section measured for collisions between singly charged xenon ions and neutral xenon atoms. ${ }^{16}$ Isotropic scattering is then assumed to determine the post-collision parameters and the subsequent sputtering. Only the ions near the walls are considered for collisions as they are the most likely to impact the walls after being scattered.

\section{Results}

Results for the base configuration are shown in Figs. 4-9. Overall, the results are satisfactory compared to the experimental data, ${ }^{17,18}$ though there is clearly room for further improvement. Figures 4 and 5 show contour plots of the plasma density at the beginning-of-life and after 1000 hours respectively. The erosion after 1000 hours is significant in comparison to the channel width and can be seen to affect the plasma contours in the thruster. Figure 6 shows the total volumetric erosion rate versus time. The erosion is no longer zero after 1000 hours, but is lower than expected at later times. However, the erosion at the exit plane, shown in Fig. 7, matches very well with experimental data. It is the upstream erosion that is not being captured correctly, and this is evident in Figs. 8 and 9, especially at later times. Overall, the extent of the curvature of the erosion profiles is not reproduced well by the model. 


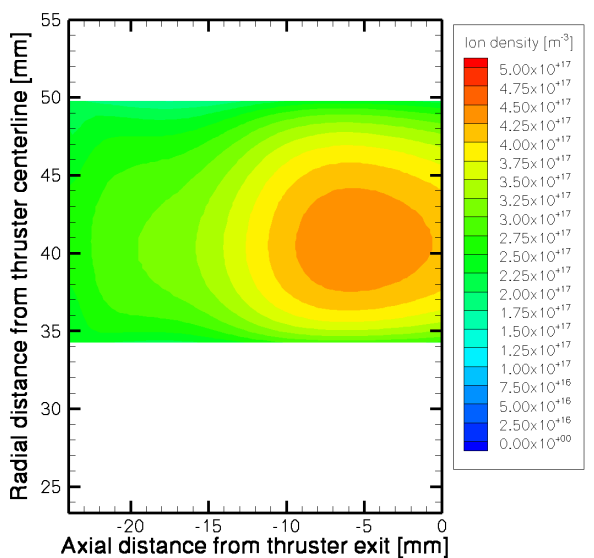

Figure 4. The ion density within the thruster at the beginning of life.

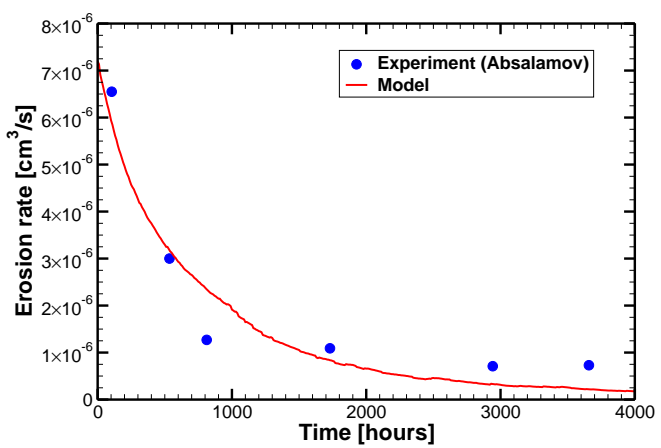

Figure 6. The total volumetric erosion rate.

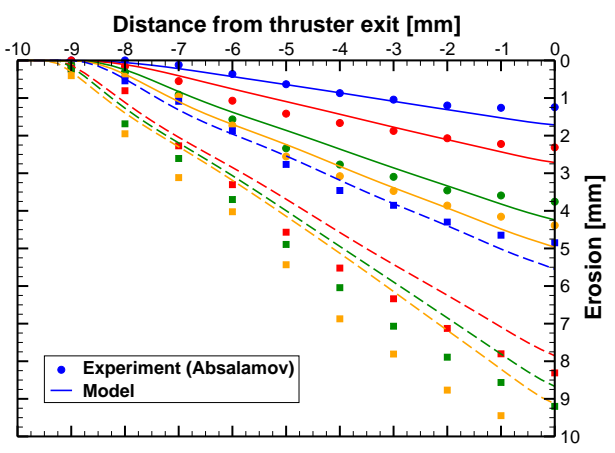

Figure 8. The inner wall erosion profiles at 160 , $310,600,800,1000,2400,3300$, and 4000 hours.

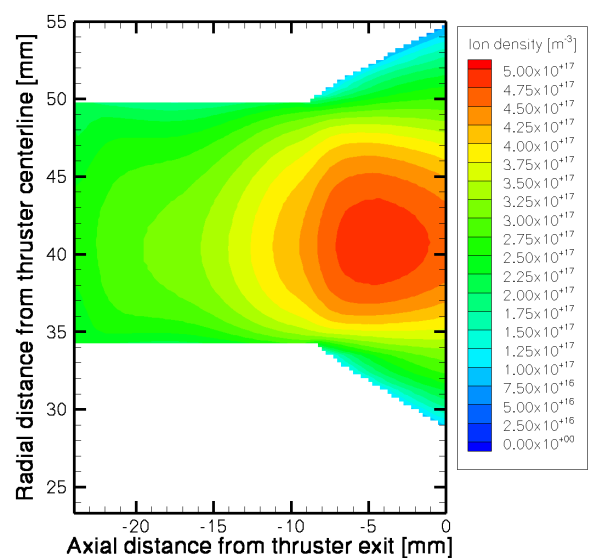

Figure 5. The ion density within the thruster after 1000 hours.

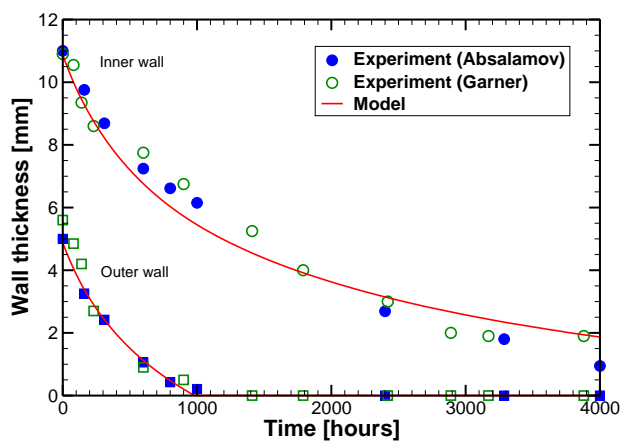

Figure 7. The wall thickness at the exit plane.

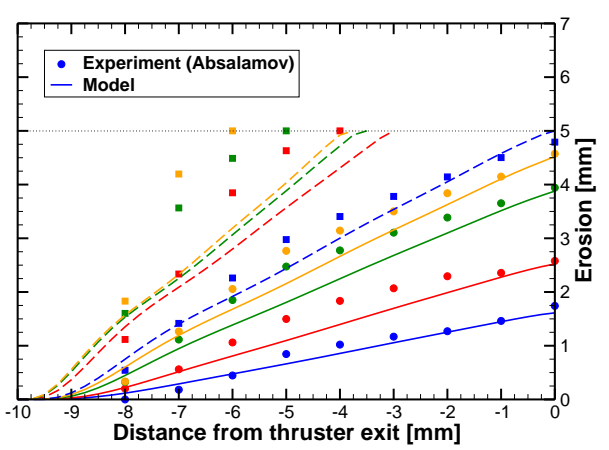

Figure 9. The outer wall erosion profiles at 160, $310,600,800,1000,2400,3300$, and 4000 hours. 
The effects of changing the ion velocity distribution are shown in Fig. 10. The initial radial erosion rates along the outer wall are shown. An ion temperature equal to the assumed neutral temperature of 1000K, or around $0.1 \mathrm{eV}$, is used in the base configuration. Cold ions as well as a $1 \mathrm{eV}$ ion temperature case are used for comparison. In general, a higher ion temperature leads to greater erosion. Overall, the effects are not large. An ion temperature of $1 \mathrm{eV}$ increases the radial erosion rate by about $3 \%$ over the $0.1 \mathrm{eV}$ case. The upstream location where erosion begins is the most affected region. The start of erosion is pushed a millimeter downstream with cold ions and several millimeters upstream with a $1 \mathrm{eV}$ temperature. The inclusion of the velocity distributions smooths the erosion profile in general. This is due to the presence of ions that now have enough energy to surpass the threshold required for sputtering.

The addition of ions diverted to the walls from elastic collisions also increases erosion as reported elsewhere, ${ }^{2}$ but for this case amounts to less than a $1 \%$ increase. This low amount is contributed in part from the fact that only the ions closest to the walls are considered for scattering. This would lead to a very small number of ions being scattered to the walls as the ion density in the near wall region tends to be the lowest within the thruster.

Figure 11 displays the effect of changing the threshold energy for sputtering. The threshold energy is increased and decreased by ten electron volts from the $60 \mathrm{eV}$ threshold used earlier in the fit to the Garnier sputter yield data. Again, the initial radial erosion rates along the outer wall are shown. Essentially, the erosion rate curves are shifted by about $35 \%$ either way. The shape of the erosion rate profiles are not affected much.

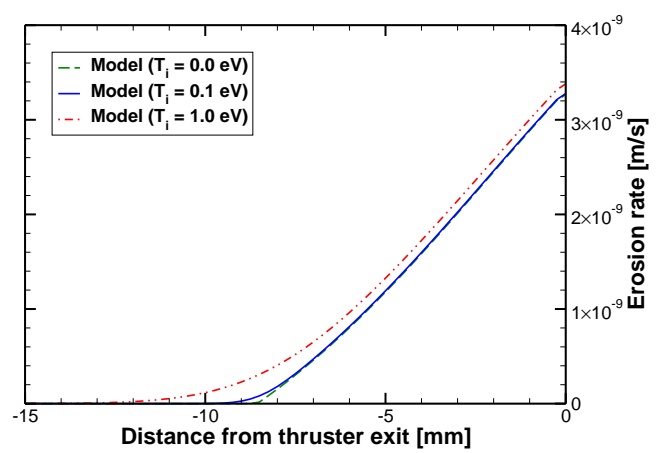

Figure 10. The initial radial erosion rates along the outer wall under different ion temperatures.

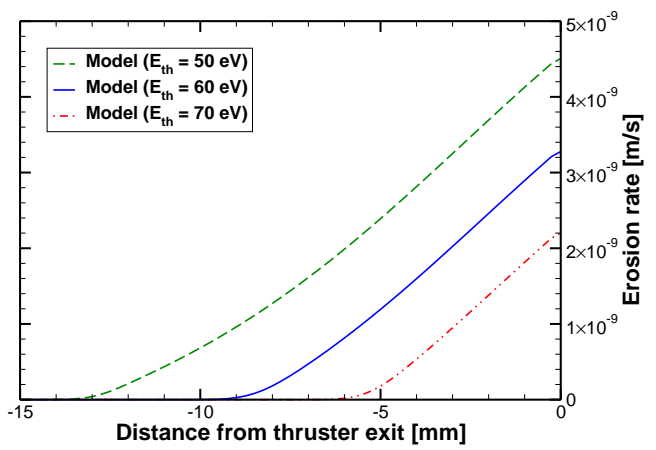

Figure 11. The initial radial erosion rates along the outer wall with different threshold energies.

Changing the sputter yield model has a significant effect on the erosion rates as seen in Fig. 12. Using Britton's sputter yield data gives an erosion rate at the exit plane that is three times greater than that calculated using Garnier's sputter yields. Gamero-Castaño's fit for $\mathrm{BNSiO}_{2}$ is also shown in comparison. Even though $\mathrm{BNSiO}_{2}$ has higher overall sputter yields than $\mathrm{BN}$, here the erosion rates at the exit plane are a quarter of those from the logarithmic fit. These differences in the magnitude of erosion rates are due to the shape of the curve fits near the threshold energy seen in Fig. 2. The logarithmic fits have a much steeper slope near the threshold energy and have concave down shape, whereas the modified Yamamura profile has a lower slope near the threshold energy and is concave up. The shape of the sputter yield curve fits may also influence the shape of the erosion profiles. Figure 13 displays the erosion along the outer wall after 160 hours for the different curve fits. The results have been normalized to the value at the exit plane given by the results of the model using the logarithmic fit on Garnier's data for easier comparison. Here, the different curvatures of the erosion profiles are more pronounced and correlate to the shape of the sputter yield curve fits near the threshold energy.

Finally, the effect of the potential drop within the thruster on the erosion is investigated. The potential is calculated from the electric field, which in turn is dependent on the electron mobility. As mentioned before, anomalous Bohm diffusion is included in the model. For the results presented above, the coefficient for the Bohm diffusion is set to $1 / 35$. As a parametric study, $1 / 16$, the classical value, and $1 / 44$, a value we have used before ${ }^{8}$ are also used. As expected, if the Bohm diffusion is decreased, the potential drop within the 


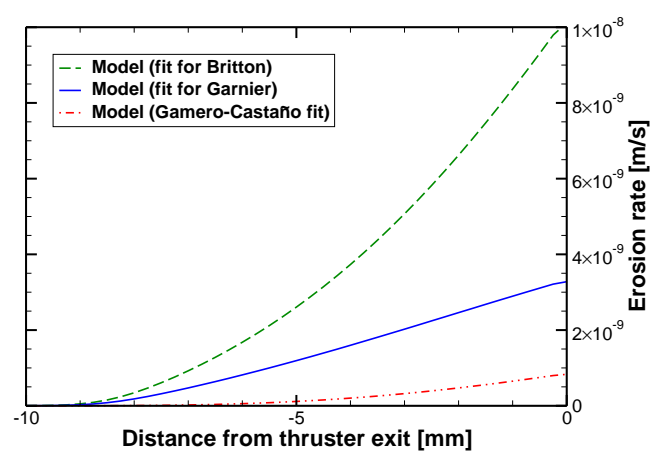

Figure 12. The initial radial erosion rates along the outer wall with different sputter yield models.

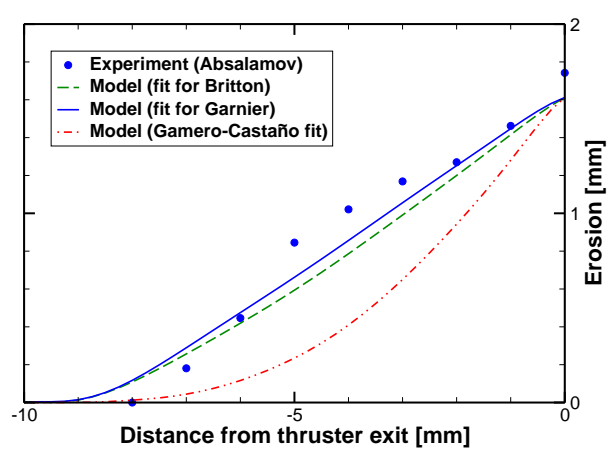

Figure 13. Normalized outer wall erosion after 160 hours with different sputter yield models.

thruster, from the anode to the exit plane, increases and vice versa as seen in Fig. 14. Figure 15 shows the resulting erosion profiles. The erosion increases as the potential drop increases due to the higher energy ions. For the lower Bohm coefficient, the potential drop within the thruster is increased by $15 \%$, but the erosion increases by $39 \%$ at the exit plane. With a higher Bohm coefficient, the potential drop decreases by $40 \%$ and the erosion decreases by $74 \%$ at the exit plane.

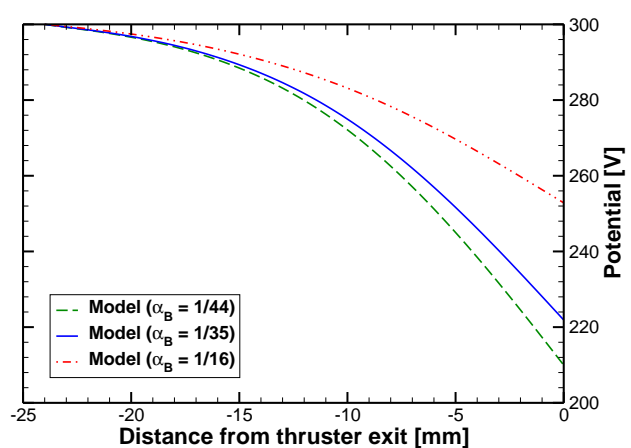

Figure 14. The potential within the thruster for different Bohm coefficients.

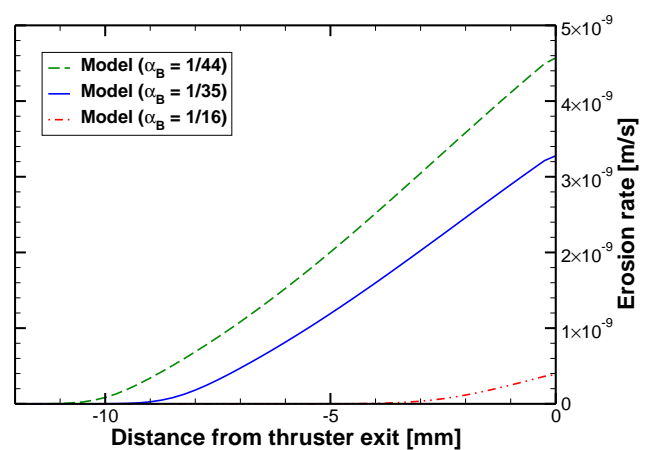

Figure 15. The initial radial erosion rates along the outer wall with different Bohm coefficients.

Another result related to the potential drop is its change over time. Figure 16 shows that the potential drop from the anode to the exit plane decreases as the walls erode away. This in turn leads to a corresponding decrease in thrust as well, seen in Fig. 17. The calculated thrust is lower than the experimentally measured values around $80 \mathrm{mN}$, but this is to be expected as the thrust is calculated at the exit plane in the model. Continued acceleration of the ions occurs beyond the exit plane due to the remaining potential drop located outside the channel. The drop in thrust of about $5 \mathrm{mN}$ from the beginning-of-life has been noted before, though it is limited to the first 1000 hours and rises a couple of $\mathrm{mN}$ after that before stabilizing. ${ }^{18-20}$

\section{Conclusion}

A hydrodynamic description of the plasma flow within a SPT-100 type Hall thruster was used to model the erosion of the channel walls over time. The simulations required time on the order of tens of minutes to run. The erosion results over time, especially at the exit plane, matched with experimental data fairly 


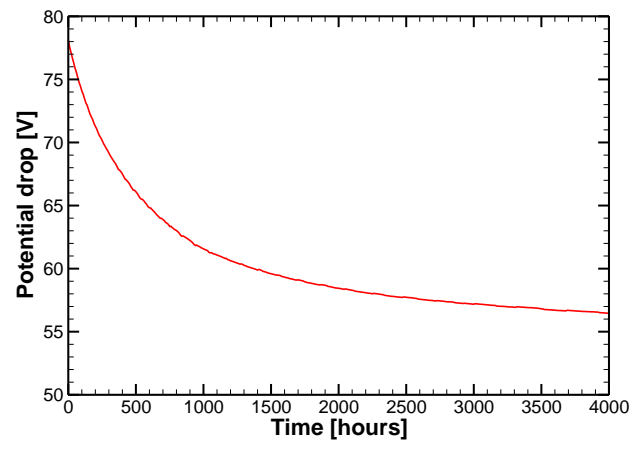

Figure 16. The calculated potential drop from the anode to the exit plane over time.

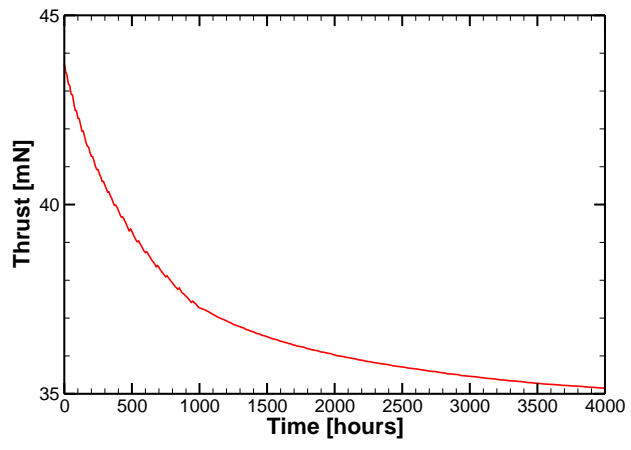

Figure 17. The thrust calculated at the exit plane over time.

well. The erosion around $5 \mathrm{~mm}$ upstream of the exit plane tended to be underpredicted, however, and the shape of the erosion profiles was not accurately captured. The overall erosion rate was lower than expected at later times.

Various factors that may play a role in the erosion process were investigated. The addition of erosion caused by ions scattered from the main flow to the walls did not significantly increase the erosion rate. It is underpredicted somewhat since only the ions closest to the walls are considered for such scattering collisions. The inclusion of velocity distributions resulting from an ion temperature also increased erosion, though it was not a drastic increase. It primarily affects the upstream portion of the erosion, since it smooths out the location where erosion begins. Changing the threshold energy shifted the erosion rate profiles. It did not change the overall shape of the profiles, however. Changing the sputter yield function near the threshold value did have a significant effect on the erosion. In particular, the slope and the curvature of the sputter yield fits affect not only the magnitude of the erosion rates, but possibly the erosion profiles as well. The results of the different threshold energies and curve fits seem to suggest that for erosion modeling efforts it is important to understand the sputtering near the threshold energy.

Finally, the erosion was a function of the potential drop within the thruster as expected. As the potential drop increased, the ion energy increased and therefore the erosion as well. The potential drop within the thruster was noted to decrease over time as the walls erode away. The change in geometry of the channel affects the plasma and thruster properties. This decrease in the potential drop led to a decrease in the thrust, which has been observed experimentally near the beginning-of-life. It also plays a role in the decrease in the erosion rate over time.

Future work on Hall thruster erosion studies will need to examine in greater detail the sputter yield model as there is much uncertainty in the low energy regions, which unfortunately is where most of the ions involved in erosion lie. Continued improvement of Hall thruster physics models will also increase fidelity of the erosion models. Although the sheath was not examined in great detail here, it certainly plays an important role in the erosion process. Continued comparisons against more experimental data, including other thrusters, will further development of erosion studies as well.

\section{Acknowledgments}

Part of this work was supported by NASA Glenn Research Center through Grant NNCO4GA56G. The first author would also like to acknowledge support from the National Science Foundation through their Graduate Research Fellowship Program.

\section{References}

${ }^{1}$ Yu, D., Li, Y., and Song, S., "Ion Sputtering Erosion of Channel Wall Corners in Hall Thrusters," Journal of Physics D: Applied Physics, Vol. 39, 2006, pp. 2205-2211. 
${ }^{2}$ Sommier, E., Allis, M. K., and Cappelli, M. A., "Wall Erosion in 2D Hall Thruster Simulations," 29th International Electric Propulsion Conference, Oct. 2005, IEPC-2005-189.

${ }^{3}$ Gamero-Castaño, M. and Katz, I., "Estimation of Hall Thruster Erosion Using HPHall," 29th International Electric Propulsion Conference, Oct. 2005, IEPC-2005-303.

${ }^{4}$ Yim, J. T., Keidar, M., and Boyd, I. D., "An Evaluation of Sources of Erosion in Hall Thrusters," 41st AIAA/SAE/ASME/ASEE Joint Propulsion Conference and Exhibit, July 2005, AIAA 2005-3530.

${ }^{5}$ Yim, J. T., Keidar, M., and Boyd, I. D., "A Hydrodynamic-Based Erosion Model for Hall Thrusters," 29th International Electric Propulsion Conference, Oct. 2005, IEPC-2005-013.

${ }^{6}$ Maslennikov, N. A., "Lifetime of the Stationary Plasma Thruster," 24th International Electric Propulsion Conference, 1995, IEPC 95-75.

${ }^{7}$ LeVeque, R. J., "Wave Propagation Algorithms for Hyperbolic Systems," Journal of Computational Physics, Vol. 131, No. 2, 1997, pp. 327-353.

${ }^{8}$ Keidar, M., Boyd, I. D., and Beilis, I. I., "Plasma Flow and Plasma-Wall Transition in Hall Thruster Channel," Physics of Plasmas, Vol. 8, No. 12, Dec. 2001, pp. 5315-5322.

${ }^{9}$ Roe, P. L., "Approximate Riemann Solvers, Parameter Vectors, and Difference Schemes," Journal of Computational Physics, Vol. 135, No. 2, 1997, pp. 250-258.

${ }^{10}$ Yang, G., Causon, D. M., Ingram, D. M., Saunders, R., and Batten, P., "A Cartesian Cut Cell Method for Compressible Flows Part A: Static Body Problems," The Aeronautical Journal, Vol. 101, 1997, pp. 47-56.

${ }^{11}$ De Zeeuw, D. and Powell, K. G., "An Adaptively Refined Cartesian Mesh Solver for the Euler Equations," Journal of Computational Physics, Vol. 104, No. 1, 1993, pp. 56-68.

${ }^{12}$ Garnier, Y., Viel, V., Roussel, J.-F., and Bernard, J., "Low-Energy Xenon Ion Sputtering of Ceramics Investigated for Stationary Plasma Thrusters," Journal of Vacuum Science and Technology A, Vol. 17, No. 6, Nov/Dec 1999, pp. 3246-3254.

${ }^{13}$ Britton, M., Waters, D., Messer, R., Sechkar, E., and Banks, B., "Sputtering Erosion Measurement on Boron Nitride as a Hall Thruster Material," Tech. rep., NASA, 2002, NASA TM-2002-211837.

${ }^{14}$ Yamamura, Y. and Tawara, H., "Energy Dependence of Ion-Induced Sputtering Yields from Monatomic Solids at Normal Incidence," Atomic Data and Nuclear Data Tables, Vol. 62, No. 2, March 1996, pp. 149-253.

${ }^{15}$ Manzella, D. H., Yim, J. T., and Boyd, I. D., "Predicting Hall Thruster Operational Lifetime," 40th AIAA/SAE/ASME/ASEE Joint Propulsion Conference and Exhibit, July 2004, AIAA 2004-3953.

${ }^{16}$ Boyd, I. D. and Dressler, R. A., "Far-Field Modeling of the Plasma Plume of a Hall Thruster," Journal of Applied Physics, Vol. 92, Aug. 2002, pp. 1764-1774.

${ }^{17}$ Absalamov, S. K. et al., "Measurement of Plasma Parameters in the Stationary Plasma Thruster (SPT-100) Plume and Its Effect on Spacecraft Components," 28th AIAA/SAE/ASME/ASEE Joint Propulsion Conference and Exhibit, July 1992, AIAA 92-3156.

${ }^{18}$ Garner, C. E., Brophy, J. R., Polk, J. E., and Pless, L. C., "Cyclic Endurance Test of a SPT-100 Stationary Plasma Thruster," 30th AIAA/SAE/ASME/ASEE Joint Propulsion Conference and Exhibit, July 1994, AIAA 94-2856.

${ }^{19}$ Arhipov, B. A. et al., "The Results of 7000-hour SPT-100 Life Testing," 24th International Electric Propulsion Conference, 1995, IEPC 95-39.

${ }^{20}$ Day, M., Maslennikov, N., Randolph, T., and Rogers, W., "SPT-100 Subsystem Qualification Status," 32nd AIAA/SAE/ASME/ASEE Joint Propulsion Conference and Exhibit, July 1996, AIAA 96-2713. 